\title{
Consideraciones anestésicas en el paciente con enfermedad por virus dengue
}

JESÚS H. TOVAR MD. ${ }^{1}$, MIGUEL A. PINZÓN MD. ${ }^{1}$, DIEGO F. RINCÓN MD. ${ }^{1}$, CARLOS E. JIMÉNEZCANIZALEZ MD. ${ }^{2}$, ÁLVARO MONDRAGÓN-CARDONA MD. ${ }^{2}$, MARTÍN E. ARRIETA-MENDOZA MD. ${ }^{3}$

\begin{abstract}
\section{Anesthetic considerations in the patient with dengue virus disease}

Dengue fever continues to be a public health problem in America. It usually presents as a mild febrile illness, however, patients with severe disease may have hemorrhagic syndromes and dengue shock. Different circumstances can arise in the evolutionary course of patients with dengue impacting on the possibility that it is necessary to perform a surgical intervention, before which it is necessary to raise the anesthetic considerations that must be taken into account for the correct approach. The present text aims to establish useful clinical considerations for anesthetic management in the patient with dengue disease to reduce the risk of peri-operative complications and death.
\end{abstract}

\section{Resumen}

El dengue continúa siendo un problema de salud pública en América. Suele presentarse como una enfermedad febril leve, sin embargo, los pacientes con enfermedad grave pueden presentar síndromes hemorrágicos y choque por dengue. Diferentes circunstancias pueden surgir en el curso evolutivo de los pacientes con dengue impactando en la posibilidad de que sea necesario realizar en ellos una intervención quirúrgica, ante lo cual es necesario plantear las consideraciones anestésicas que deben ser tenidas en cuenta para el correcto abordaje. El presente texto tiene como objetivo establecer consideraciones clínicas útiles para el manejo anestésico en el paciente con enfermedad por dengue para reducir el riesgo de complicaciones peri-operatorias y muerte.
Key words: Dengue, severe dengue, anesthesia, Colombia.

\section{Palabras clave:}

Dengue, dengue grave, anestesia, Colombia.

\footnotetext{
Departamento de anestesiología y reanimación. Hospital Universitario Hernando Moncaleano Perdomo, Universidad Surcolombiana. Neiva, Huila, Colombia.

2 Departamento de Medicina Interna. Hospital Universitario Hernando Moncaleano Perdomo, Universidad Surcolombiana. Neiva, Huila, Colombia.

3 Universidad de Sucre, Sincelejo, Sucre, Colombia.
}

\section{ORCID}

https://orcid.org/0000-0001-5577-5434 


\section{Introducción}

$\mathrm{E}$ I dengue es una enfermedad febril aguda transmitida por vector del género Aedes (mosquitos Aedes aegypti y Aedes albopictus)[1], transcurre en la gran mayoría de los casos como una enfermedad leve caracterizada por fiebre, mialgias, artralgias y cefalea retro ocular, que mejora sin intervención ni complicación alguna, sin embargo, se pueden desarrollar formas graves por extravasación de plasma, shock, hemorragias importantes y compromiso multisistémico que pueden llevar rápidamente al enfermo a la muerte, por lo que se hace importante detectar, clasificar y tratar al enfermo con dengue, sobretodo en el ámbito hospitalario en un paciente con posible intervención quirúrgica.

Esta infección viral cobra importancia a nivel mundial ya que ha sido la enfermedad tropical con mayor crecimiento en las ultimas 3 décadas, siendo vigilado y monitorizado por la OMS, quien calcula una incidencia anual aproximada de 50 millones de casos por dengue, con una tasa de mortalidad para las américas que ronda el 1,2\%[1], teniendo Colombia la mayor cantidad de casos y mortalidades de los países andinos, con un $75 \%$ de los casos, presentándose con menos frecuencia hacia los países del cono sur, donde el mayor afectado es Brasil con el $65 \%$ de los casos[2].

La enfermedad es producida por uno de los cuatro serotipos del virus dengue (DENV1 a DENV4), cuya estructura está compuesta básicamente por una envoltura, un genoma de ARN, un precursor de membrana, 3 proteínas estructurales y 7 proteínas no estructurales (NS)[3]. Una vez el virus es inoculado por el vector en los capilares sanguíneos, ocurre un periodo de incubación de 5 a 7 días en promedio. Este ingresa a las células dendríticas de la piel por medio de endocitosis mediada por receptores, las cuales migran a los ganglios linfáticos, y órganos hematopoyéticos, donde se da la replicación viral con viremia, activándose la respuesta inmune a través de células $B, T C D 4$ y CD8, citosinas inflamatorias como el IFN y respuesta humoral a través de la formación de anticuerpos de tipo IgM e lgG específicos contra la proteína no estructural NS-1. El recuento plaquetario puede descender progresivamente desde la etapa febril, pero este descenso se hace más intenso en la etapa crítica, la trombocitopenia no es debida a un déficit de producción sino a la destrucción masiva periférica, por un mecanismo inmunomediado (anticuerpos antivirales con reacción cruzada contra las plaquetas). El incremento de la permeabilidad vascular se debe a la inflamación endotelial producida principalmente por una proteína del dengue llamada NS - 1 desencadenando una liberación exagerada de citocinas pro inflamatorias que aumentan la permeabilidad vascular. Además, la infección endotelial por el dengue induce apoptosis y aumento en la producción de matriz metaloproteinasa que incrementa aún más la permeabilidad vascular[7],[8].

Es importante reconocer que la mayoría de las infecciones por DENV (80\%), suelen ser asintomáticas. Cuando se presentan síntomas existe un amplio espectro de presentaciones clínicas que varían desde síndromes febriles indiferenciados hasta casos graves que requieren manejo en $\mathrm{UCl}$. Debido a la variedad en la presentación la OMS y con el fin de dar manejo acorde al grado de compromiso de los pacientes, se clasifica la enfermedad en dos grandes grupos; Dengue y Dengue grave, a su vez el Dengue se clasifica en base a los síntomas presentados en; Dengue sin signos de alarma o Dengue con signos de alarma (Tabla 1). Los signos de alarma son hallazgos clínicos predictores de un peor desenlace clínico y evolución a dengue grave, sin llegar a ser criterios de gravedad[2] (Tabla 2).

\begin{tabular}{lll} 
& \multicolumn{2}{c}{ Tabla 1. Clasificación Dengue OMS } \\
\hline \multicolumn{2}{c}{ Dengue } & \multicolumn{1}{c}{ Dengue grave } \\
Fengue sin signos de alarma & \multicolumn{1}{c}{ Dengue con signos de alarma } & \multicolumn{1}{c}{ Extravasación grave de plasma } \\
gias, cefalea & Dolor abdominal intenso, vomito per- Sangrado importante \\
& sistente, acumulación clínica de liqui- \\
& dos, Sangrado de mucosas, Letargia, \\
& agitación \\
& Hepatomegalia $>2 \mathrm{~cm}$ \\
& Laboratorio: aumento del hematocrito \\
& concurrente con rápida disminución \\
& del número de plaquetas & \\
& & Compromiso organico grave \\
\hline
\end{tabular}




\section{Tabla 2. Criterios de gravedad Dengue. OMS}

Extravasación grave de plasma $\quad \begin{gathered}\text { Sangrado importante } \\ \text { - Shock }\end{gathered}$
- Acumulación de líquidos con insufi-
ciencia respiratoria

\section{Compromiso orgánico grave}

- Hígado: AST o ALT 1.000

- Sistema nervioso central: Alteración de la conciencia (letargo o agitación, coma, convulsiones), Encefalitis

- Corazón: miocarditis por dengue

- Renal: Insuficiencia renal aguda
Posterior al periodo de incubación del virus, se inicia la etapa febril de la enfermedad (3 a 5 días) que coincide con la viremia, donde se observan síntomas acompañantes a la fiebre más comunes como cefalea, artralgias, mialgias, astenia, dolor abdominal, emesis y exantema, siendo más raro la diarrea, odinofagia y síntomas respiratorios (sobre todo en niños). Hay que prestar especial atención a las manifestaciones hemorrágicas como epistaxis, petequias, gingivorragia, o pruebas de fragilidad capilar positivas (test del torniquete). A la exploración física es común la presencia de hepatomegalia, y se dirige a descartar complicaciones de extravasación capilar. Las ayudas diagnósticas van enfocadas a confirmar el diagnóstico, clásicamente se ha realizado demostrando la presencia de IgM Dengue después del $5^{\text {to }}$ día del inicio de la fiebre, sin embargo, ante episodios graves, rápidamente evolutivos, se puede aplicar la prueba rápida dengue para detectar el antígeno NS1, O PCR para detectar el genoma del virus. Otros laboratorios importantes en el paciente con sospecha de dengue es el hemograma completo (en especial el recuento plaquetas, hemoglobina y hematocrito), así como AST, ALT, tiempos de coagulación, pruebas de función renal. Otras ayudas como la ecografía abdominal total, radiografía de tórax preferiblemente en decúbito lateral derecho buscan aparición de tercer espacio por extravasación de plasma o la evidencia de colecistitis acalculosa. Las pruebas más específicas como troponina, punción lumbar se reservan para casos graves específicos[4]. Es importante recalcar que las manifestaciones graves, y shock por dengue generalmente aparece una vez cede la fiebre y hay una aparente mejoría del cuadro, este período se conoce como etapa crítica y puede dar evolución a manifestaciones de alarma o gravedad de dengue porque es el periodo donde existe mayor permeabilidad capilar, o a la etapa de resolución de la enfermedad.

No existe tratamiento específico para la infección, según su gravedad se indica el manejo, que se basa principalmente en la administración abundante de líquidos, en los casos sin signos de alarma estos líquidos se pueden administrar vía oral, se puede manejar ambulatoriamente con acetaminofén para la fiebre y el dolor, así como medidas físicas antitérmicas y vigilancia de aparición de signos de alarma. No es necesario el estudio más allá de un recuento plaquetario para vigilancia de plaquetas y hematocrito en los casos leves sin signos de alarma. Cuando los signos de alarma se presentan, es recomendable hospitalizar para instaurar manejo con cristaloides intravenosos, toma de paraclínicos correspondientes, y vigilancia estrecha en busca de manifestaciones hemorrágicas o extravasación de plasma. Para los casos graves es importante el manejo intensivo, así como la vigilancia hemodinámica estrecha, según la manifestación que presente el paciente (miocarditis, encefalitis, hepatitis, shock), administración de líquidos intravenosos en cantidad suficiente para compensar la fuga de plasma, soporte inotrópico, o ventilación mecánica si requiere. Es importante restringir el consumo de ASA o AINES ya que facilitan las hemorragias, de igual importancia es vigilar el descenso del hematocrito, y el gasto urinario, debido a que en la etapa de recuperación se puede desencadenar sobrecarga hídrica por la redistribución de plasma, que puede producir complicaciones como edema agudo de pulmón[2].

Generalmente, la enfermedad leve evoluciona hacia la resolución espontánea, sin mayor intervención a mantener un adecuado estado de hidratación y vigilancia de los signos de alarma para intervenir y disminuir la mortalidad por dengue. El presente texto tiene como objetivo establecer consideraciones clínicas útiles para el manejo anestésico en el paciente con enfermedad por dengue para reducir el riesgo de complicaciones peri-operatorias y muerte. Siendo Colombia y los países andinos una zona endémica, por lo cual no es raro que el anestesiólogo se vea enfrentado a un paciente con dengue que requiera cirugía de manera electiva o urgente[5],[6]. 


\section{Consideraciones anestésicas en el paciente con Dengue}

Cuando un paciente presenta un evento clínico que posiblemente lo lleve a una intervención quirúrgica, y por ende un procedimiento anestésico, en el desarrollo de una enfermedad febril aguda con diagnóstico de dengue, se debe tener en cuenta la extravasación de líquidos, hemorragia o daño de algún órgano. Según la etapa clínica en la que se encuentre la enfermedad, puede evolucionar a un cuadro de distrés respiratorio, así como presentar complicaciones tales como hemorragias masivas, falla orgánica multisistémica y coagulación intravascular diseminada que dificultan el enfoque anestésico. Se debe vigilar la presión arterial diferencial de $20 \mathrm{~mm} \mathrm{Hg}$ o menos ya que cuando no se hace diagnóstico temprano de shock la mortalidad puede llegar hasta el 30\%.

El sangrado es una de las principales complicaciones en el dengue aumentando la morbilidad y mortalidad, se presenta más frecuentemente entre el cuarto y quinto día de la enfermedad (etapa crítica). La patogénesis de la hemorragia es multifactorial, e incluye vasculopatía, deficiencia plaquetaria y defectos en los factores de la coagulación. Las hemorragias más comunes incluyen epistaxis, sangrados en piel y tracto gastrointestinal los cuales son sitios intervenidos directamente por el anestesiólogo. La incidencia de estas hemorragias se ha reportado entre un 5 - $8 \%$ en diferentes series de casos.

En el dengue también se presenta compromiso inmunológico debido a que el virus produce leucopenia caracterizada principalmente por linfopenia con neutrofilia leve relativa. Esta linfopenia contribuye al retraso en la eliminación del virus, al aumento de la carga viral y teóricamente al desarrollo de complicaciones. Estas características sumadas a la posible depresión del sistema inmune que puede provocar la anestesia general hace pensar en el desarrollo de infecciones relacionadas con la cirugía, sin embargo, en la literatura no se encuentra reportado el aumento de esta complicación.

Los pacientes con dengue pueden ser llevados a cirugía por las mismas causas que la población general, sin embargo, existen estudios que reportan una mayor frecuencia de patologías que se derivan en eventos quirúrgicos (Tabla 3)[9].

La complicación más común de estos eventos es la hemorragia, por lo cual es necesario instaurar una terapia hídrica adecuada y realizar transfusiones de glóbulos rojos, plaquetas, plasma fresco congelado y crioprecipitado para restaurar la hemostasia y la estabilidad hemodinámica de manera oportuna antes de la inducción anestésica e intervención quirúrgica. Además algunas de estas patologías como la colecistitis y la pancreatitis se pueden solucionar con tratamiento médico basados en una buena hidratación y corrección de la trombocitopenia[10].

\section{Evaluación preoperatoria}

La evaluación clínica del paciente con dengue debe ser lo más detallada posible, y se deben registrar los ítems evaluados en la historia clínica preoperatoria, prestando especial atención a la presencia de los signos de alarma de dengue, o a la posibilidad de que ya se haya desarrollado una forma grave de éste. (Tabla 2), así mismo se considerarán las demás variables en la evaluación pre anestésicas del paciente como riesgo quirúrgico, posibilidad de una vía aérea difícil y estado hemodinámico.

En cuanto a los paraclínicos se recomienda solicitar en todos los pacientes con signos de alarma hemograma completo, transaminasas, tiempos de coagulación, ionograma, creatinina, BUN, albúmina, glicemia, radiografía de tórax (Descartar derrame pleural por dengue) y electrocardiograma (descartar miocarditis por dengue)[19].

Se debe establecer la clasificación del riesgo anestésico, la clase funcional, corregir las alteraciones detectadas antes de llevar el paciente a cirugía y planear

\begin{tabular}{lc}
\multicolumn{2}{c}{$\begin{array}{c}\text { Tabla 3. Eventos quirúrgicos más frecuentes en } \\
\text { pacientes con dengue }\end{array}$} \\
\hline $\begin{array}{l}\text { Evento Quirúrgico } \\
\text { Colecistitis acalculosa }\end{array}$ & Frecuencia \\
Pancreatitis aguda & $5,26 \%$ \\
Abscesos abdominales & $2,17 \%$ \\
Hemorragia de tracto digestivo & $1,60 \%$ \\
superior & $1,48 \%$ \\
Apendicitis aguda & $0,46 \%$ \\
Síndrome compartimental de & $0,41 \%$ \\
antebrazo & \\
Hemorragia cerebral & $0,34 \%$ \\
Hematoma de pared abdomi- & $0,34 \%$ \\
nal anterior & $0,23 \%$ \\
Síndrome compartimental & \\
abdominal & $0,23 \%$ \\
Hematoma retroperitoneal & $0,11 \%$ \\
\hline Ruptura esplénica espontánea & \\
\hline
\end{tabular}


el acto anestésico con base en los parámetros clínicos y paraclínicos. Siendo necesario posponer toda cirugía electiva en pacientes con dengue.

Es importante tener en cuenta la importancia de hacer un abordaje clínico inicial del paciente con dengue para hacer un enfoque terapéutico adecuado, recomendamos la clasificación hemodinámica descrita por la Organización Mundial de Salud, en la cual se clasifica el paciente siguiendo criterios clínicos en estable, choque compensado y choque severo por dengue[19].

En cuanto a los medicamentos antiplaquetarios en pacientes con dengue se suspenden, ya que en el caso del ácido acetil salicílico que es el medicamento más estudiado se ha demostrado aumento del riesgo de sangrado mayor. La excepción es en pacientes con angioplastia reciente (menor de un mes) que deben continuar la terapia a pesar de la infección por dengue. Todos los pacientes con dengue requieren profilaxis con omeprazol para hemorragia del tracto digestivo superior[11],[12].

\section{Transoperatorio}

En pacientes con trombocitopenia menor de 100.000 $\mathrm{mm}^{3}$, no se recomienda anestesia neuroaxial por el alto riesgo de hematoma espinal. La anestesia general es una elección más segura en estos casos.

Para la intubación se debe preparar el equipo de vía aérea difícil, ya que estos pacientes pueden presentar edema de la vía aérea y tendencia al sangrado[13]. Se debe hacer monitorización no invasiva estricta con electrocardiografía, medición de presión arterial, pulsioximetría, temperatura (mantener mayor de $37^{\circ} \mathrm{C}$ ), diuresis $(1-2 \mathrm{cc} / \mathrm{kg} / \mathrm{h})$ y medición de $\mathrm{CO}_{2}$ al final de la espiración.

En la inducción anestésica se debe tener especial cuidado en mantener la estabilidad cardiovascular. Durante la intubación evitar una respuesta hipertensiva que aumente la presión intracerebral y por lo tanto, el riesgo de hemorragia cerebral. En los reportes de series de casos recomiendan el uso de midazolam, fentanilo, tiopental o propofol y relajación con rocuronio o vecuronio. El mantenimiento de la anestesia lo realizaron con isorane o sevoflorane más fentanilo o remifentanilo[14].

Durante el perioperatorio los pacientes pueden presentar complicaciones respiratorias intrínsecas por la afectación del virus, pero también por la terapia hídrica agresiva. Estas se pueden presentar en cualquier momento del perioperatorio, con mayor incidencia en el período postoperatorio. Cuando se presenta síndrome de distres respiratorio el paciente debe ser soportado con oxigenoterapia $\mathrm{FIO}_{2} 100 \%$ y ventilación mecánica.

Es importante considerar durante el acto anestésico que el choque por dengue se caracteriza por hipotensión debido a disminución de la resistencia vascular periférica, y cardiodepresión (bradicardia relativa), por lo cual el manejo se debe hacer tanto con expansores de volumen como con inotrópicos. De los fluidos evaluados la solución Hartman ha demostrado los mejores resultados en este escenario. El cálculo de líquidos basales es de 2 - $3 \mathrm{ml} / \mathrm{kg} / \mathrm{h}$. Si el paciente se encuentra con signos de shock se administra bolos de $10-20 \mathrm{ml} / \mathrm{kg}$ y se continúa la expansión de volumen con $10 \mathrm{ml} / \mathrm{kg} / \mathrm{h}$ evaluando de una manera dinámica el cambio en los signos vitales, la diuresis y los cambios en el hematocrito. Si no hay mejoría con los bolos de líquidos se debe iniciar soporte vasopresor con noradrenalina[15],[16],[17].

La causa de las hemorragias es multifactorial. Se debe a trombocitopenia, combinada con vasculopatía y disfunción hepática que a su vez conduce a coagulación intravascular diseminada. Hay que sospechar sangrado interno significativo en pacientes con signos de hipovolemia sin elevación del hematocrito[20], en tales circunstancias es necesaria la transfusión sanguínea, monitorizando la su respuesta clínica. Por otro lado, es importante resaltar que la transfusión de plaquetas no ha demostrado ser efectiva en la prevención y el control del sangrado, pero puede ser beneficiosa en pacientes con hemorragia activa y trombocitopenia severa (menos de $10.000 / \mathrm{mm}^{3}$ ) [21],[22]. También es indicado administrar vitamina $\mathrm{K}$ en pacientes con tiempo de protrombina prolongado y disfunción hepática severa[23].

El manejo del sangrado debe ser entonces según la magnitud del sangrado con glóbulos rojos, plaquetas $(0,2-0,4 \mathrm{u} / \mathrm{kg})$, plasma fresco congelado $(20 \mathrm{ml} /$ $\mathrm{kg})$ y crioprecipitado $(0,2-0,4 \mathrm{u} / \mathrm{kg})[18],[10]$.

\section{Analgesia}

El dolor postoperatorio debe ser manejado de manera eficiente siguiendo como principio general que no se debe administrar ningún medicamento por vía intramuscular a los pacientes con dengue. Los antiinflamatorios no esteroideos están contraindicados, ya que aumentan el riesgo de sangrado. Se puede considerar el uso de dipirona en centros de alta complejidad, pero no en pacientes[1] pediátricos. El manejo del dolor se hace con base en Acetaminofén $1 \mathrm{~g}$ cada 6 horas en adultos y $10-15 \mathrm{mg} / \mathrm{kg}$ cada 6 horas en 
niños. Se puede combinar con opioides como la morfina en las dosis convencionales.

No se debe administrar bloqueos regionales a pacientes con recuentos de plaquetas menores de $60.000[12],[17],[18]$.

\section{Postoperatorio}

Los pacientes que fueron llevados a cirugía con dengue grave requieren seguir manejo en unidad de cuidados intensivos, con monitorización continua y balance estricto de líquidos. Además, se debe mantener un pH mayor de 7,2. En los reportes de casos citados los pacientes con dengue llevados a cirugía muestran mayor riesgo de sangrado postoperatorio, pero no tuvieron mayor disminución de plaquetas comparados con el resto de pacientes con dengue.
Tampoco se encontró aumento en las infecciones del sitio quirúrgico[5],[6],[14].

\section{Conclusiones}

En base a todo lo expuesto, es evidente que debido a la frecuencia de la enfermedad en las américas, asociado a los cambios climáticos y la movilización masiva de pasajeros entre los países sudamericanos hacia áreas tropicales endémicas, es necesario el conocimiento fisiopatológico, la adecuada exploración y valoración pre anestésica de pacientes con cuadro febriles agudos compatibles con dengue, que necesitan ser llevados a cirugías urgentes, ya que el manejo temprano y la corrección de anomalías en la homeostasis antes, durante y posterior al acto anestésico, redundara en buen desenlace clínico del paciente en el perioperatorio.

\section{Referencias}

1. OMS. Dengue and severe dengue. Factsheet No 117, April 2017. World Health Organization, 2017.

2. OMS. Dengue: guías para el diagnóstico, tratamiento, prevención y control. World Health Organization, 2009.

3. Chambers TJ, Hahn CS, Galler $\mathrm{R}$, Rice CM. Flavivirus genome organization, expression, and replication. Annu Rev Microbiol. 1990;44:649-88.

4. Botero D, Restrepo M. Infecciones en la práctica médica. Dengue. Cap 93.

5. Shamim M. Frequency, pattern and management of acute abdomen in dengue fever in Karachi, Pakistan. Asian J Surg. 2010;33(3):107113. doi:10.1016/S10159584(10)60019-X.

6. McFarlane MEC, Plummer JM, Leake PA, et al. Dengue fever mimicking acute appendicitis: A case report. Int J Surg Case Rep. 2013;4(11):1032-1034. doi:10.1016/j.ijscr.2013.08.017.
7. Oishi K, Saito M, Mapua CA, Natividad FF. Dengue illness: Clinical features and pathogenesis. J Infect Chemother. 2007;13(3):125-133. doi:10.1007/s10156-007-05169.

8. Chiu $Y C$, Wu KL, Kuo $\mathrm{CH}$, et al. Endoscopic findings and management of dengue patients with upper gastrointestinal bleeding. Am J Trop Med Hyg. 2005;73(2):441-444.

9. Ahmad F, Surgeon V, Hospital $H L$, et al. Management Experience of Surgical Complications of Dengue Fever Patients at Hameed Latif Hospital, Lahore. 2013;19(1):49-54.

10. Chuansumrit A, Chaiyaratana W. Hemostatic derangement in dengue hemorrhagic fever. Thromb Res. 2014;133(1):1016. doi:10.1016/j. thromres.2013.09.028.

11. DENGUE GCP GUIDELINES 2012. Acad Med Malaysia. 2012.

12. Malagon JN, Padilla JC, Rojas-Alvarez DP. Guía de Atención Clínica Integral del paciente con Dengue. Infectio. 2011;15(4):293-
301. doi:10.1016/S01239392(11)70744-5.

13. Chhabra A, Malhotra N. Anesthetic management of a pregnant patient with dengue hemorrhagic fever for emergency cesarean section. Int J Obstet Anesth. 2006;15(4):306-310. doi:10.1016/j.ijoa.2006.04.011.

14. Rawat SKS, Mehta $Y$, Juneja R, Trehan N. Dengue fever in a patient recovering from coronary artery bypass grafting. Ann Card Anaesth. 14(2):155-156.

15. Ngo NT, Cao XT, Kneen R, et al. Acute management of dengue shock syndrome: a randomized double-blind comparison of 4 intravenous fluid regimens in the first hour. Clin Infect Dis. 2001;32(2):204-213. doi:10.1086/318479.

16. Wills BA, Dung NM, Loan HT, et al. Comparison of Three Fluid Solutions for Resuscitation in Dengue Shock Syndrome. World Health. 2005:877-889. doi:10.1056/NEJMoa1404304.

17. ENFERMEDADES INFECCIOSAS, DENGUE. GUIA PARA EL EQUIPO DE SALUD. Minist salud, Argenti- 
na. 2013.

18. Lothar A Schwarte, Jenny Vieveen EP and PS. Anesthetic implications of dengue, Abstrac. Br J Anaesth. 2012;108(suppl 2):ii215-ii277. doi:10.1093/bja/ aer485.

19. https://www.cdc.gov/dengue/ resources/dengue-clinicianguide_508.pdf

20. WHO (2009). Dengue: guidelines for diagnosis, treatment, prevention and control - new edition.
WHO, Geneva 2009

21. Lack of efficacy of prophylactic platelet transfusion for severe thrombocytopenia in adults with acute uncomplicated dengue infection. Lye DC, Lee VJ, Sun Y, Leo YS Clin Infect Dis. 2009 May;48(9):1262-5.

22. Prospective observational study of low thresholds for platelet transfusion in adult dengue patients.Thomas L, Kaidomar S, Kerob-Bauchet B, Moravie V,
Brouste $Y$, King JP, Schmitt $S$, Besnier F, Abel S, Mehdaoui H, Plumelle $Y$, Najioullah F, Fonteau C, Richard P, Césaire R, CabiéA Transfusion. 2009;49(7):1400.

23. WHO Regional Office for Southeast Asia. Comprehensive guidelines for prevention and control of dengue and dengue haemorrhagic fever. Revised and expanded version. SEARO Technical Publication Series, New Delhi 2011. 